\section{Fatores associados à realização dos exames de rastreamento para o câncer de próstata: um estudo de base populacional}

\author{
Factors associated with prostate cancer screening: \\ a population-based study
}

1 Faculdade de Ciências Médicas, Universidade Estadual de Campinas, Campinas, Brasil.

2 Faculdade de Saúde Pública, Universidade de São Paulo, São Paulo, Brasil.

3 Faculdade de Medicina, Universidade de São Paulo, São Paulo, Brasil. ${ }^{4}$ Faculdade de Medicina, Universidade Estadual Paulista, Botucatu, Brasil. 5 Secretaria de Estado da Saúde de São Paulo, São Paulo, Brasil.

Correspondência V.M.S. L.Amorim Departamento de Medicina Preventiva e Social, Faculdade de Ciências Médicas,

Universidade Estadual de Campinas.

Rua Tessália Vieira de Camargo 126, Campinas, SP 13083-887, Brasil.

vivimaelamorim@gmail.com

\section{Abstract}

The aim of this study was to analyze the prevalence of prostrate cancer screening among men aged 50 years or older based on socioeconomic, demographic, and health-related behavioral variables and the presence of morbidity. A population-based cross-sectional study was performed. The following factors were associated with failure to undergo screening: age under 70 years; less than eight years of schooling; per capita household income less than one-half the minimum wage; not having diabetes; lack of visual impairment; and lack of visit to the dentist in the previous year. The Brazilian public healthcare system accounted for $41 \%$ of the reported prostate cancer screening tests. According to the present study, despite controversy over the effectiveness of digital rectal examination and prostrate-specific antigen for detecting prostate cancer, a significant portion of the male population has been undergoing these tests, the access to which displays significant socioeconomic inequalities.

Men's Heealth; Mass Screening; Prostatic Neoplasms
Vivian Mae Schmidt Lima Amorim 1

Marilisa Berti de Azevedo Barros 1

Chester Luiz Galvão César 2

Moisés Goldbaum 3

Luana Carandina 4

Maria Cecília Goi Porto Alves 5

\section{Introdução}

O câncer de próstata é o segundo câncer em incidência entre os homens no mundo e a quinta causa de mortalidade por tumores malignos entre os homens, a exceção do câncer de pele nãomelanoma ${ }^{1}$. No Brasil, após o câncer de pele, o de próstata é o que apresenta maior incidência, sendo a quarta causa de morte por neoplasias nos homens 2. Para o ano de 2010, no país, estimou-se a incidência de 54 casos novos por 100 mil homens 2 .

O câncer de próstata tem crescimento lento, é raro antes dos 50 anos de idade, sendo que $85 \%$ dos casos são diagnosticados após os 65 anos e a sua historia natural ainda é pouco conhecida 3,4 .

Até o momento, somente alguns marcadores ou fatores de riscos foram identificados como idade, raça/etnia e a história familiar deste câncer em pai ou irmão 5,6. Pesquisas sobre outros possíveis fatores de risco, como ingestão de gorduras, consumo de álcool, tabagismo e vasectomia, têm apresentado resultados contraditórios 3,6. Alguns estudos apontaram a ingestão de licopeno, encontrado nos tomates e produtos derivados, bem como a ingestão de selênio como fatores de proteção para o câncer de próstata, mas também são relatadas controvérsias sobre este assunto 8,9.

O rastreamento do câncer de próstata é realizado por meio do toque retal e da dosagem do Antígeno Específico Prostático (PSA). O toque retal é utilizado para avaliar o tamanho, a forma 
e a consistência da próstata no sentido de verificar a presença de nódulos, mas sabe-se que este exame apresenta algumas limitações, uma vez que somente possibilita a palpação das porções posterior e lateral da próstata, deixando $40 \%$ a $50 \%$ dos tumores fora do seu alcance; depende também do treinamento e experiência do examinador e ainda existe a resistência e rejeição de parcela importante dos pacientes em relação a esse tipo de exame 10,11.

O PSA é uma glicoproteína originária na próstata, e o seu nível elevado na corrente sangüínea é considerado um importante marcador biológico para algumas doenças da próstata, entre elas, o câncer 4,12,13. O antígeno prostático é produzido pelas células epiteliais da próstata e não especificamente pela célula cancerosa, podendo também estar alterado em outras patologias e resultar na realização de biópsias desnecessárias 4,12,13.

Estudos internacionais apresentam opiniões contraditórias sobre o uso do rastreamento, e alguns apontam que essa prática pouco tem contribuído para o declínio na taxa de mortalidade por esse agravo 14,15,16. Observa-se, então, que frente a essas questões não existe consenso entre as sociedades científicas internacionais em relação aos benefícios e riscos na utilização de programas de rastreamento populacional para o câncer de próstata 17,18,19.

No Brasil, o Ministério da Saúde também não recomenda o rastreamento populacional para o câncer de próstata, mas enfatiza que o homem que venha a se submeter aos exames, por meio rastreamento oportunístico ou por livre demanda, seja previamente orientado sobre os benefícios, os riscos e limitações dos exames para que a partir destas informações possa tomar a decisão de realizar ou não o exame 2,20 .

Apesar da existência de polêmica sobre o assunto, em vários países e também no Brasil, questões referentes ao uso do toque retal e principalmente do PSA passaram a fazer parte de inquéritos populacionais de saúde para avaliar as prevalências da realização destes exames e os fatores associados à realização dos exames de detecção precoce do câncer de próstata. Estudos feitos com base nesses inquéritos têm identificado alguns fatores associados à realização dos exames, como a idade 21,22, o nível socioeconômico $23,24,25$, raça/grupos étnicos $23,24,25$, e o estado conjugal 23,25,26.

Considerando a carência que existe no Brasil de informações de base populacional sobre a prevalência da realização de exames de detecção do câncer de próstata, a polêmica existente quanto à realização dos exames, buscou-se com o presente estudo analisar os fatores associados à realização dos exames de rastreamento para este agravo em municípios do Estado de São Paulo, no sentido de contribuir para o conhecimento das ações de prevenção e controle do câncer de próstata que vêm sendo executadas.

\section{Métodos}

\section{População de estudo}

Este é um estudo transversal de base populacional desenvolvido com os dados do Inquérito Multicêntrico de Saúde no Estado de São Paulo (ISA$\mathrm{SP})$, realizado em alguns municípios do estado, no período entre 2001 e 200227.

$\mathrm{O}$ inquérito teve como base uma amostra probabilística, estratificada, por conglomerados e obtida em dois estágios de seleção. No primeiro, os setores censitários das áreas selecionadas foram agrupados em três estratos, segundo o percentual de chefes de família com nível universitário: menos de $5 \%$, de $5-24,9 \%$ e $25 \%$ ou mais, visando a aumentar a probabilidade de que indivíduos pertencentes aos estratos de maior nível socioeconômico da população fossem incluídos na amostra. Foram sorteados dez setores de cada estrato, de cada área estudada, com probabilidade proporcional ao tamanho, por meio de sorteio sistemático aplicado à relação dos setores ordenados segundo tipo de setor dentro de cada estrato 27 .

No segundo estágio da amostragem foram selecionados os domicílios, também por meio de sorteio sistemático aplicado às relações de domicílios existentes em cada setor sorteado. A definição das pessoas que deveriam ser entrevistadas em cada domicílio obedeceu à probabilidade de encontrar 250 pessoas de cada domínio de sexo e idade.

Foram definidos domínios de idade e sexo em relação aos quais era desejável obter um tamanho mínimo de amostra. Esses domínios foram: $<1$ ano, 1-11 anos, 12-19 masculino, 12-19 feminino, 20-59 masculino, 20-59 feminino, 60 e mais masculino, e 60 e mais feminino. Estimou-se um tamanho mínimo de amostra de 192 indivíduos para cada domínio de idade e sexo previamente definido, tendo como base a estimativa de prevalência de $50 \%$ com nível de $95 \%$ de confiança, erro máximo de 0,10 e efeito do delineamento (deff) igual a 2. Considerando-se os possíveis efeitos da não-resposta (casa fechada e recusa) ao redor de $20 \%$, o tamanho da amostra foi corrigido para 250 indivíduos em cada domínio 27 .

Para o presente estudo foram utilizados os dados referentes a todos os homens com 50 anos ou mais ( $\mathrm{n}=992)$, não institucionalizados, resi- 
dentes nas quatro áreas do Estado de São Paulo incluídas no inquérito (Município de Campinas, Município de Botucatu, a administração regional do Butantã no Município de São Paulo e uma área formada pelos municípios de Taboão da Serra, Embu e Itapecerica da Serra).

As informações foram obtidas por entrevistadores treinados, que aplicaram um questionário estruturado em 19 blocos temáticos, com a maioria das questões fechadas. As questões de interesse específico para este estudo referem-se a características socioeconômicas do entrevistado e da família, realização de exames preventivos para o câncer de próstata, presença de doenças crônicas autorreferidas, deficiência física, comportamentos relacionados à saúde, saúde emocional e autoavaliação da saúde.

A variável dependente analisada foi ter realizado o toque retal e/ou PSA em algum momento da vida. Quanto a esses exames, foi também analisado o tipo de exame realizado (toque retal e PSA), há quanto tempo foi feito e se o pagamento foi particular ou pelo SUS.

As variáveis independentes analisadas foram:

- Socioeconômicas e demográficas: idade, raça/ cor (autorreferida), naturalidade, município de moradia, situação conjugal, escolaridade, renda familiar mensal per capita, situação ocupacional, religião, número de pessoas no domicílio e posse de bens duráveis;

- Comportamentos relacionados à saúde: prática de atividade física em contexto de lazer pelo menos uma vez por semana, situação tabágica (fumante, não fumante e ex-fumante), frequência semanal de consumo de álcool e dependência alcoólica pelo teste de Cage 28 ;

- Relacionadas ao estado de saúde: número de doenças crônicas referidas, presença de limitação física, transtorno mental comum avaliado pelo Self Reporting Questionnaire (SRQ-20) sendo usado como ponto de corte para o transtorno mental comum 6 ou mais respostas positivas 29 , o índice de massa corporal $\left(\mathrm{IMC}=\mathrm{kg} / \mathrm{m}^{2}\right)$ calculado com base no peso e altura referidos;

- Relacionadas ao uso de serviços de saúde: consulta odontológica no ano que antecedeu a entrevista e vacinação contra gripe (para os homens com 60 anos ou mais de idade).

Os dados do inquérito foram digitados em banco elaborado com o programa Epi Info 6.04b (Centers for Disease Control and Prevention, Atlanta, Estados Unidos) e submetido à análise de consistência. Para as análises estatísticas realizadas neste estudo foi utilizado o programa Stata 9.0 (Stata Corp., College Station, Estados Unidos) que possibilita considerar as variáveis do plano complexo de amostragem: estratos, conglomera- dos e ponderações. As análises bivariadas incluíram estimativas de prevalência e intervalos de 95\% de confiança (IC95\%) da "não realização de algum exame preventivo para o câncer de próstata" segundo as variáveis socioeconômicas, de comportamentos relacionados à saúde e estado de saúde. O modelo final foi realizado por meio de regressão de Poisson com variância robusta, e para entrada no modelo múltiplo foram consideradas as variáveis independentes que apresentaram valor de p inferior a 0,20 na análise bivariada, tendo permanecido no modelo final as variáveis que apresentavam $\mathrm{p}<0,05$.

O projeto de pesquisa foi aprovado pelo Comitê de Ética da Faculdade de Ciências Médicas da Universidade Estadual de Campinas, sob parecer 369/2000.

\section{Resultados}

No presente estudo foram analisados os dados de 992 homens com 50 anos ou mais de idade. Dentre eles, predominaram os de 60 a 69 anos $(51,7 \%)$, de cor autorreferida branca (77\%), com cônjuge (76,3\%), com menos de 9 anos de escolaridade $(76,4 \%)$ e renda familiar per capita menor que 2,5 salários mínimos (59\%).

Verificou-se que 44,4\% (IC95\%: 37,3-51,7) da população estudada nunca haviam realizado exame preventivo para o câncer de próstata. A não realização de exames preventivos para o câncer de próstata foi significativamente mais prevalente nos homens com menos de 70 anos, nos de raça/cor preta ou parda, naqueles com escolaridade menor que 9 anos, nos residentes na cidade de Botucatu, nos que não exercem a chefia familiar, com renda familiar per capita menor que 2,5 salários mínimos, nos desempregados e naqueles com menor posse de bens duráveis (Tabela 1).

Dos homens que referiram ter realizado exames de detecção do câncer de próstata (55,6\%), o toque retal foi referido por $61,8 \%$, o PSA por $73,2 \%$, a ultrassonografia por $28,2 \%$ e a biópsia por 7,3\%. Foi observada a concomitância da realização de dois ou mais exames: $22 \%$ realizaram toque retal e PSA, cerca de $18 \%$ realizaram toque retal, PSA e ultrassonografia ou biópsia e 9,8\% referiram a realização dos quatro exames. Ocorreram ainda outras associações de exames diferentes das referidas (11,3\%). Dentre os homens que referiram ter realizado algum exame, $49,7 \%$ haviam feito no ano que antecedeu a entrevista, $23 \%$ entre um e dois anos, $10 \%$ entre dois e três anos, e $17 \%$ quatro anos ou mais antes da entrevista. Dos exames realizados, $41 \%$ foram financiados pelo SUS. Cerca de $1 \%$ dos 
Prevalência da não realização de exames preventivos para câncer de próstata, segundo variáveis socioeconômicas e demográficas em homens de 50 anos ou mais. Inquérito Multicêntrico de Saúde no Estado de São Paulo (ISA-SP), 2001-2002.

\begin{tabular}{|c|c|c|c|c|}
\hline Variáveis & $n$ * & $\begin{array}{c}\text { Prevalência em \% } \\
\text { (IC95\%) }\end{array}$ & RP (IC95\%) & Valor de $p$ \\
\hline Idade (em anos) & & $0,0355 * *$ & & \\
\hline $50-59$ & 144 & $48,5(37,9-59,3)$ & $1,13(1,04-1,23)$ & 0,004 \\
\hline $60-69$ & 504 & $43,0(35,6-50,7)$ & $1,09(1,01-1,17)$ & 0,018 \\
\hline 70 ou mais & 344 & $30,7(24,1-38,2)$ & 1,00 & \\
\hline Cor/raça *** & & $0,0085 * *$ & & \\
\hline Branca & 771 & $39,3(31,7-47,5)$ & 1,00 & \\
\hline Preta/Parda & 186 & $63,2(47,3-76,8)$ & $1,17(1,04-1,30)$ & 0,005 \\
\hline Escolaridade (em anos) & & $0,0016 * \star$ & & \\
\hline $0-8$ & 742 & $50,8(43,5-50,8)$ & $1,17(1,06-1,29)$ & 0,001 \\
\hline 9 ou mais & 246 & $28,4(18,2-41,6)$ & 1,00 & \\
\hline Local de moradia & & $0,2826 * *$ & & \\
\hline Butantã & 212 & $41,0(29,4-53,8)$ & 1,00 & \\
\hline Campinas & 256 & $41,6(30,9-53,1)$ & $1,00(0,88-1,13)$ & 0,953 \\
\hline Municípios da grande São Paulo & 278 & $52,7(38,2-66,8)$ & $1,08(0,94-1,23)$ & 0,233 \\
\hline Botucatu & 246 & $58,6(50,0-66,7)$ & $1,12(1,01-1,24)$ & 0,029 \\
\hline Naturalidade & & $0,3055 * \star$ & & \\
\hline Próprio município & 191 & $34,5(22,1-49,5)$ & 1,00 & \\
\hline Outros municípios de São Paulo & 404 & $44,7(32,7-57,3)$ & $1,07(0,94-1,22)$ & 0,281 \\
\hline Outros estados & 394 & $49,1(39,3-58,9)$ & $1,74(0,98-1,24)$ & 0,085 \\
\hline Situação conjugal & & $0,1798 * \star$ & & \\
\hline Casado/União estável & 821 & $43,0(35,2-51,1)$ & 1,00 & \\
\hline Solteiro/Separado/Desquitado/Viúvo & 170 & $54,1(40,0-64,5)$ & $1,07(0,96-1,20)$ & 0,172 \\
\hline Religião & & $0,5105 * \star$ & & \\
\hline Católica & 741 & $42,3(34,7-50,3)$ & 1,00 & \\
\hline Outras & 107 & $51,3(32,8-69,3)$ & $1,06(0,92-1,22)$ & 0,382 \\
\hline Evangélicas & 132 & $51,5(31,7-70,8)$ & $1,06(0,92-1,22)$ & 0,374 \\
\hline Situação na família & & $0,0210 * \star$ & & \\
\hline Chefe & 929 & $42,8(35,7-50,3)$ & 1,00 & \\
\hline Não chefe & 63 & $70,7(46,6-86,9)$ & $1,19(1,04-1,36)$ & 0,008 \\
\hline Renda familiar per capita (salários mínimos) & & $0,0027 \star \star$ & & \\
\hline$\leq 0,5$ & 100 & $80,1(65,5-89,5)$ & $1,39(1,23-1,57)$ & 0,000 \\
\hline $0,5-1,0$ & 150 & $55,6(41,2-69,2)$ & $1,20(1,04-1,37)$ & 0,009 \\
\hline $1,0-2,5$ & 329 & $47,7(35,7-60,1)$ & $1,14(1,00-1,29)$ & 0,039 \\
\hline $2,5-4,0$ & 163 & $38,6(24,3-55,2)$ & $1,07(0,92-1,23)$ & 0,346 \\
\hline$\geq 4,0$ & 250 & $29,5(18,7-43,2)$ & 1,00 & \\
\hline Situação ocupacional & & $0,0079 * *$ & & \\
\hline Aposentado & 502 & $38,4(28,7-49,1)$ & 1,00 & \\
\hline Trabalha & 427 & $43,5(34,7-52,7)$ & $1,03(0,95-1,13)$ & 0,412 \\
\hline Desempregado & 63 & $76,6(53,3-90,4)$ & $1,27(1,12-1,45)$ & 0,000 \\
\hline Posse de bens duráveis & & $0,0158 * \star$ & & \\
\hline $0-6$ & 334 & $55,1(43,1-66,6)$ & $1,16(1,03-1,30)$ & 0,011 \\
\hline $7-9$ & 269 & $50,9(38,5-63,1)$ & $1,12(1,01-1,25)$ & 0,029 \\
\hline $10-15$ & 386 & $33,6(24,1-44,7)$ & 1,00 & \\
\hline
\end{tabular}

IC95\%: intervalo de 95\% de confiança; RP: razão de prevalências.

* $\mathrm{N}=992$;

** Valor de p;

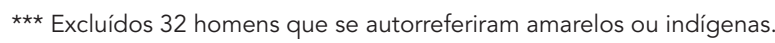


entrevistados referiu ter no momento ou já ter tido o câncer de próstata (dados não apresentados em tabela).

Quanto aos comportamentos relacionados à saúde, observou-se significativa maior prevalência de realização dos exames de rastreamento de câncer de próstata entre os homens que haviam feito consulta odontológica no último ano (Tabela 2).

Quanto às morbidades, foi observado que os homens que referiram ter diabetes apresentaram maior prevalência de realização dos exames em relação aos que referiram não ter a doença. Verificou-se maior prevalência de não realização dos exames preventivos nos homens que referiram ter deficiência visual (Tabela 3).

Os resultados da análise de regressão múltipla de Poisson apontam que a "não realização" dos exames preventivos para o câncer de próstata foi significativamente mais frequente nos homens com menos de 70 anos, menor escolaridade, de menor renda familiar per capita, que não apresentam diabetes mellitus, que referiram algum tipo de deficiência visual e que não fizeram consulta odontológica no ano que antecedeu a entrevista (Tabela 4).

\section{Discussão}

A utilização de exames de rastreamento do câncer de próstata é cercada por controvérsias na comunidade científica internacional, existindo diferentes recomendações das sociedades médicas e agências governamentais. Estudos realizados sobre o rastreamento do câncer de próstata

Prevalência da não realização de exames preventivos para câncer de próstata, segundo comportamentos relacionados à saúde em homens de 50 anos ou mais. Inquérito Multicêntrico de Saúde no Estado de São Paulo (ISA-SP), $2001-2002$.

\begin{tabular}{|c|c|c|c|c|}
\hline Variáveis & $\mathbf{n}$ & $\begin{array}{c}\text { Prevalência em } \\
\text { \% (IC95\%) }\end{array}$ & $\begin{array}{l}\text { RP bruta } \\
\text { (IC95\%) }\end{array}$ & Valor de $p$ \\
\hline Hábito de fumar & & $0,7121 *$ & & \\
\hline Não fumante & 330 & $44,3(33,5-55,7)$ & 1,00 & \\
\hline Ex-fumante & 449 & $41,9(31,9-52,7)$ & $0,98(0.89-1,07)$ & 0,717 \\
\hline Fumante & 210 & $48,5(35,9-61,4)$ & $1,02(0,91-1,15)$ & 0,622 \\
\hline Consumo de álcool (vezes por semana) & & 0,4107 & & \\
\hline Não & 447 & $40,2(29,8-51,5)$ & 1,00 & \\
\hline 1 ou mais & 406 & $44,1(35,1-53,5)$ & $1,02(0,93-1,12)$ & 0,558 \\
\hline Menos de 1 & 123 & $52,8(36,9-68,1)$ & $1,08(0,96-1,23)$ & 0,171 \\
\hline Cage & & 0,8431 * & & \\
\hline Negativo & 921 & $44,2(37,0-51,5)$ & 1,00 & \\
\hline Positivo & 71 & $46,6(24,9-69,6)$ & $1,01(0,86-1,20)$ & 0,843 \\
\hline Prática de exercícios físicos & & 0,6283 * & & \\
\hline Não pratica & 643 & $46,7(37,9-55,8)$ & 1,00 & \\
\hline Pratica & 84 & $37,8(20,3-59,2)$ & $0,93(0,80-1,09)$ & 0,422 \\
\hline Só caminhadas & 265 & $41,0(28,0-55,7)$ & $0,96(0,85-1,07)$ & 0,494 \\
\hline $\operatorname{IMC}\left(\mathrm{kg} / \mathrm{m}^{2}\right)$ & & 0,5337 * & & \\
\hline$<25$ & 470 & $47,4(38,7-56,3)$ & $1,05(0,96-1,15)$ & 0,232 \\
\hline $25-29$ & 340 & $39,4(28,2-51,9)$ & 1,00 & \\
\hline$\geq 30$ & 119 & $40,6(23,0-61,0)$ & $1,00(0,86-1,17)$ & 0,912 \\
\hline Consultas odontológicas no último ano & & $0,0029 *$ & & \\
\hline $\operatorname{Sim}$ & 347 & $33,0(23,5-44,1)$ & 1,00 & \\
\hline Não & 645 & $54,1(45,2-62,7)$ & $1,15(1,05-1,27)$ & 0,002 \\
\hline Vacina contra Influenza no último ano ** & & 0,4283 * & & \\
\hline $\operatorname{Sim}$ & 536 & $38,1(31,8-44,9)$ & 1,00 & \\
\hline Não & 297 & $41,8(32,8-51,4)$ & $1,02(0,96-1,09)$ & 0,429 \\
\hline
\end{tabular}

IC95\%: intervalo de 95\% de confiança; IMC: índice de massa corporal; RP: razão de prevalências.

* Valor de $\mathrm{p}$;

** Homens com 60 anos ou mais. 
Prevalência da não realização de exames preventivos para câncer de próstata, segundo morbidade em homens de 50 anos ou mais. Inquérito Multicêntrico de Saúde no Estado de São Paulo (ISA-SP), 2001-2002.

\begin{tabular}{|c|c|c|c|c|}
\hline Variáveis & $\mathrm{n}$ & $\begin{array}{c}\text { Prevalência em \% } \\
\text { (IC95\%) }\end{array}$ & RP bruta IC $95 \%$ & Valor de $p$ \\
\hline Presença de doenças crônicas & & 0,3210 & & \\
\hline Nenhuma & 166 & $53,1(37,0-68,5)$ & $1,11(0,97-1,27)$ & 0,125 \\
\hline $1-2$ & 424 & $44,5(34,3-55,2)$ & $1,05(0,93-1,18)$ & 0,411 \\
\hline 3 ou mais & 374 & $37,6(26,0-50,8)$ & 1,00 & \\
\hline Diabetes mellitus & & 0,000 * & & \\
\hline Sim & 134 & $17,3(10,6-26,9)$ & 1,00 & \\
\hline Não & 853 & $47,7(39,7-55,8)$ & $1,25(1,15-1,37)$ & 0,000 \\
\hline Hipertensão arterial sistêmica & & 0,1656 * & & \\
\hline $\operatorname{Sim}$ & 386 & $38,7(29,0-49,3)$ & 1,00 & \\
\hline Não & 601 & $48,3(38,9-57,8)$ & $1,06(0,97-1,17)$ & 0,164 \\
\hline Limitação física & & $0,0187 *$ & & \\
\hline Não & 619 & $42,7(34,4-51,6)$ & 1,00 & \\
\hline Auditiva & 141 & $29,7(17,7-45,2)$ & $0,90(0,79-1,03)$ & 0,139 \\
\hline Visual & 188 & $58,2(44,0-71,2)$ & $1,10(1,00-1,22)$ & 0,040 \\
\hline Outras & 44 & $67,5(39,1-87,1)$ & $1,17(0,99-1,37)$ & 0,053 \\
\hline Transtorno mental comum & & 0,6797 * & & \\
\hline Sim & 207 & $41,9(28,8-56,3)$ & 1,00 & \\
\hline Não & 743 & $45,0(37,3-52,9)$ & $1,02(0,92-1,13)$ & 0,680 \\
\hline
\end{tabular}

IC95\%: intervalo de 95\% de confiança; RP: razão de prevalências.

* Valor de $p$.

mostraram-se discordantes quanto à eficácia do procedimento, dividindo a opinião dos pesquisadores e apontando a necessidade de maior investigação sobre o assunto 4,13,16.

Inquéritos de saúde vêm incorporando questões referentes à realização de exames de diagnóstico precoce do câncer de próstata, principalmente sobre o PSA, detectando a difusão da realização do exame 23,24,25.

A escolha da faixa etária estudada (50 anos ou mais de idade) considerou os dados científicos que apontam o aumento da incidência do câncer de próstata a partir desta idade 3,6,30. Estudo realizado na cidade de São Paulo com o objetivo de verificar a presença do adenocarcimona de próstata em população assintomática encontrou prevalência ascendente de $1,3 \%$ na faixa de 50 59 anos, a 5,2\% na mais idosa (70 anos ou mais), mostrando o crescimento de risco deste câncer com a idade mais avançada 31 .

A presente pesquisa permitiu verificar que 44,4\% (IC95\%: 37,3-51,7\%) dos homens com 50 anos ou mais, residentes nas quatro áreas incluídas no estudo, nunca haviam realizado toque retal ou PSA. Esse achado se assemelha aos encontrados em estudos realizados na cidade de Belo
Horizonte, Minas Gerais (46,3\%) e nos Estados Unidos (46,3\%) 21,22. Também mostra que apesar de não haver o rastreamento populacional instituído no Brasil, parcela expressiva da população masculina já realizou os exames (55,6\%), ressaltando-se que cerca de $50 \%$ dos homens que referiram ter realizado o exame o fizeram no ano prévio à entrevista.

Quanto à idade, observou-se que a realização dos exames se mostrou mais prevalente na faixa etária de 70 anos ou mais, em comparação às faixas etárias inferiores, assemelhando-se a achados de outros estudos que também verificaram que a chance de realizar um exame cresce com a idade 21,22. O aumento da prevalência e de comorbidades com o avanço da idade torna mais freqüente a procura pelos serviços de saúde, aumentando a oportunidade para a realização de exames preventivos. Além disso, com a idade cresce a incidência de hiperplasia de próstata, o que pode demandar a realização do toque retal e da dosagem do PSA.

Estudos realizados nos Estados Unidos encontraram como motivo para a não realização dos exames de PSA e toque retal a falta de conhecimento sobre a existência e a importância 
Modelo de regressão múltipla de Poisson para a não realização dos exames preventivos para o câncer de próstata em homens de 50 anos ou mais. Inquérito Multicêntrico de Saúde no Estado de São Paulo (ISA-SP), 2001-2002.

\begin{tabular}{|c|c|c|c|c|}
\hline Variáveis & $n$ * & $\mathrm{RP} * \star$ & IC95\% & Valor de $p$ \\
\hline \multicolumn{5}{|l|}{ Idade (em anos) } \\
\hline 70 ou mais & 344 & 1,00 & & \\
\hline $60-69$ & 504 & 1,14 & $1,06-1,21$ & 0,000 \\
\hline $50-59$ & 144 & 1,21 & $1,12-1,30$ & 0,000 \\
\hline \multicolumn{5}{|c|}{ Escolaridade (em anos) } \\
\hline 9 ou mais & 246 & 1,00 & & \\
\hline $0-8$ & 742 & 1,10 & $0,99-1,22$ & 0,050 \\
\hline \multicolumn{5}{|c|}{ Renda familiar per capita (salários mínimos) } \\
\hline$\leq 0,5$ & 100 & 1,25 & $1,10-1,42$ & 0,000 \\
\hline $0,5-1,0$ & 150 & 1,08 & $0,94-1,24$ & 0,250 \\
\hline $1,0-2,5$ & 329 & 1,05 & $0,92-1,20$ & 0,423 \\
\hline $2,5-4,0$ & 163 & 0,99 & $0,87-1,14$ & 0,974 \\
\hline$>4,0$ & 250 & 1,00 & & \\
\hline \multicolumn{5}{|c|}{ Diabetes mellitus } \\
\hline $\operatorname{Sim}$ & 134 & 1,00 & & \\
\hline Não & 853 & 1,26 & $1,14-1,38$ & 0,000 \\
\hline \multicolumn{5}{|l|}{ Limitação física } \\
\hline Não & 619 & 1,00 & & \\
\hline Auditiva & 141 & 0,93 & $0,81-1,08$ & 0,389 \\
\hline Visual & 188 & 1,10 & $1,01-1,20$ & 0,017 \\
\hline Outras & 44 & 1,17 & $0,99-1,37$ & 0,052 \\
\hline \multicolumn{5}{|c|}{ Consultas odontológicas no último ano } \\
\hline $\operatorname{Sim}$ & 347 & 1,00 & & \\
\hline Não & 645 & 1,14 & $1,06-1,23$ & 0,000 \\
\hline
\end{tabular}

IC95\%: intervalo de 95\% de confiança; RP: razão de prevalências.

* $\mathrm{N}=992$;

** Ajustada pelo modelo de Poisson para as demais variáveis do modelo.

dos exames, a não recomendação médica para realizá-los, o esquecimento, o medo e a vergonha em relação ao toque retal, e o receio dos resultados 11,26 .

O presente estudo apontou que homens do segmento de menor nível socioeconômico, avaliado pela renda familiar per capita e pela escolaridade, tiveram menor prevalência da realização dos exames de rastreamento para o câncer de próstata, o que se assemelha a resultados encontrados em estudos realizados nos Estados Unidos e Canadá, onde também foi observada menor prevalência de realização de PSA nos homens com menor renda e menor escolaridade $22,23,24,25,26$. Os achados no Brasil decorrem em parte pela ausência da recomendação de rastreamento no SUS e possível maior indicação do exame por médicos em clínicas privadas.

Este estudo verificou ainda que os homens que referiram ter diabetes mellitus apresentaram maior prevalência de realização dos exames preventivos para o câncer de próstata em relação àqueles que não referiram a doença. Essa associação poderia decorrer da maior freqüência de visitas ou consultas dos pacientes diabéticos aos serviços de saúde e consequente maior exposição à oferta dos exames. Além disso, estudo aponta o diabetes mellitus como um fator de risco para a hiperplasia benigna da próstata, cujos sintomas devem aumentar a probabilidade da realização do toque retal e de PSA 32 . Embora existam evidências da associação do diabetes mellitus com vários tipos de câncer, como o de pâncreas e o de vias biliares, entre outros, estas não existem em relação ao câncer de próstata 33,34.

Outro achado deste estudo foi que os homens que relataram algum tipo de deficiência visual apresentaram menor prevalência da realização dos exames. Pesquisa realizada na Califórnia, Estados Unidos, verificou que homens 
com deficiências físicas tiveram menor prevalência de realização do PSA 35. Dependendo do grau da limitação física, a necessidade total ou parcial de um cuidador para as atividades diárias poderia comprometer o acesso aos serviços de saúde.

Foi observada associação entre consulta odontológica e a realização dos exames para detecção do câncer de próstata. O uso de serviços odontológicos no ano prévio poderia ser um indicador de conduta propensa à busca a cuidados de saúde, o que levaria também à demanda de outros exames preventivos. Outros estudos têm identificado como preditor negativo do PSA o baixo acesso aos serviços de saúde e à realização de outras práticas preventivas 23,24 . A baixa procura pelos serviços de saúde reduziria a possibilidade de realização do rastreamento oportunístico para o câncer de próstata.

Na análise bivariada foi observada a associação, que não persistiu no modelo final, da cor autorreferida com a realização do toque retal e do PSA. Alguns estudos desenvolvidos nos Estados Unidos encontraram menor prevalência de realização dos exames em homens não brancos e, os autores, considerando os achados de maior risco do câncer de próstata na população negra, alertam quanto à atenção que os serviços de saúde necessitam dedicar a este segmento da população 22,23 .

O estado conjugal também se mostrou associado na análise bivariada, sem que a significância estatística persistisse no modelo final. Alguns estudos têm apontado menor prevalência de realização dos exames de rastreamento para o câncer de próstata em homens sem cônjuges 23,25. Esse achado tem sido atribuído ao incentivo que os homens receberiam de suas companheiras para cuidar da saúde 36 .

Um dado interessante observado neste estudo é que, apesar de não haver indicação pelo Ministério da Saúde do rastreamento populacional e do oportunístico para o câncer de próstata, o SUS foi responsável pela realização de $41 \%$ dos exames referidos e que possivelmente podem ter sido indicados pela presença de sintomas ou por interesse de pessoas assintomáticas. Mesmo não sendo possível determinar o real motivo da realização dos exames, considerando que esses dados não foram levantados na pesquisa, verificou-se ser o SUS um importante financiador dos exames de detecção precoce do câncer de próstata. O sistema complementar de saúde por sua vez foi o responsável majoritário pela oferta dos exames de rastreamento do câncer de próstata (59\%), o que pode apontar para uma maior flexibilidade na indicação por este segmento.

Em relação às limitações deste estudo, uma das mais importantes é o fato de a informação sobre a realização dos exames preventivos para o câncer de próstata ter sido obtida por entrevista e, portanto, estar sujeita aos vieses de memória e de informação. O entrevistado pode dizer que realizou os exames por considerar esta resposta adequada e esperada. Resultados de estudos apontam a existência de diferenças significativas entre a informação referida da realização dos exames de detecção do câncer de próstata e a existente nos prontuários médicos 37,38 , mas observa-se na literatura cientifica que esse método de obtenção de informação, por meio de inquéritos epidemiológicos, tem sido amplamente utilizado como forma de monitorar o conhecimento e execução, por parte da população, das recomendações sobre práticas preventivas para vários tipos de câncer 39 .

Outra limitação deste estudo é que o inquérito utilizado abrangeu uma temática ampla, sem maior detalhamento das questões relativas aos exames de rastreamento do câncer de próstata, tais como: se o exame feito era para prevenção, diagnóstico ou seguimento da doença, o motivo e as condições clínicas que levaram à realização dos exames e sobre o conhecimento dos resultados por parte de quem o realizou. O desenho transversal da pesquisa, por sua vez, limita a possibilidade de interpretar as associações encontradas como derivadas de relações causa-efeito.

A realização de outros estudos de base populacional sobre o tema é necessária para avaliar se os homens têm conhecimento acerca dos exames de rastreamento para o câncer de próstata e se estão sendo suficientemente informados, pelos serviços de saúde e por seus médicos, sobre os benefícios e riscos relacionados à realização destes exames e sob que circunstâncias estão sendo realizados. Constitui um desafio para os gestores das políticas de saúde a implementação das metas instituídas na Política Nacional de Atenção Integral à Saúde do Homem 40, como forma de promover e incentivar a prática de cuidados com a saúde na população masculina, e assim possibilitar o aumento da expectativa de vida e a redução dos índices de morbimortalidade por causas preveníveis e evitáveis nessa população. 


\section{Resumo}

O objetivo deste estudo foi analisar a prevalência da realização dos exames de rastreamento para o câncer de próstata em homens com 50 anos ou mais de idade, segundo variáveis socioeconômicas, demográficas, de comportamentos relacionados à saúde e presença de morbidade. O estudo foi do tipo transversal, de base populacional, e as análises estatísticas consideraram o delineamento da amostra. Os fatores associados à não realização dos exames de rastreamento do câncer de próstata, foram: ter de idade menor que 70 anos, ter escolaridade de até 8 anos, renda familiar per capita menor que 0,5 salário mínimo, não ter diabetes, ter limitação visual e não ter ido ao dentista no último ano. O SUS foi responsável pela realização de $41 \%$ dos exames de rastreamento do câncer de próstata referidos. Este estudo apontou que apesar da controvérsia sobre e efetividade do toque retal e da dosagem do Antígeno Específico Prostático (PSA) para a detecção do câncer de próstata, parcela significativa da população masculina vem realizando estes exames para os quais existem significativas desigualdades socioeconômicas quanto ao acesso.

Saúde do Homem; Programas de Rastreamento; Neoplasias da Próstata

\section{Colaboradores}

V. M. S. L. Amorim realizou a proposta do artigo, a revisão da literatura, a análise dos dados e a redação do artigo. M. B. A. Barros orientou a proposta do artigo, a análise dos dados e a redação do artigo, elaborou os instrumentos e coordenou a pesquisa de campo, bem como desenvolveu o projeto ISA-SP. C. L. G. César, M. Goldbaum, L. Carandina e M. C. G. P. Alves desenvolveram o projeto ISA-SP, elaboraram os instrumentos, coordenaram a pesquisa de campo e contribuíram na revisão do artigo.

\section{Agradecimentos}

À Fundação de Amparo à Pesquisa do Estado de São Paulo (FAPESP) - Programa de Políticas Públicas, processo nº. 88/14099-7, e à Secretaria Estadual de Saúde de São Paulo, pelo financiamento do ISA-SP. À Secretaria de Vigilância em Saúde do Ministério da Saúde, pelo suporte à análise por intermédio do Centro Colaborador em Análise de Situação de Saúde do Departamento de Medicina Preventiva e Social da Faculdade de Ciências Médicas, Universidade Estadual de Campinas.

\section{Referências}

1. Ferlay J, Shin HR, Bray F, Forman D, Mathers C, Parkin DM. Globocan 2008, cancer incidence and mortality worldwide. Lyon: International Agency for Research on Cancer; 2010. (IARC CancerBase, $10)$.

2. Instituto Nacional de Câncer. Estimativa da incidência e mortalidade por câncer no Brasil - 2008. http://www.inca.gov.br/estimativa/2008 (acessado em 27/Nov/2008).

3. Brawley OW, Ankerst DP, Thompson IM. Screening for prostate cancer. CA Cancer J Clin 2009; 59: 264-73.

4. Carroll P, Albertsen PC, Greene K, Babaian RJ, Carter HB, Gann PH, et al. Prostate-specific antigen best practice statement: 2009 update. http:// www.auanet.org/content/guidelines-and-qualitycare/clinical-guidelines/main-reports/psa09.pdf (acessado em 29/Set/2010).

5. Crawford ED. Epidemiology of prostate cancer. Urology 2003; 62 Suppl 6A:3-12.
6. Damber JE, Aus G. Prostate cancer. Lancet 2008; 371:1710-21.

7. Bryant RJ, Hamdy FC. Screening for prostate cancer: an update. Eur Urol 2008; 53:37-44.

8. Giovannuci E. Tomato products, licopene and prostate cancer: a review of the epidemiological literature. J Nutr 2005; 135:2030S-1.

9. Lippman SM, Klein EA, Goodman PJ, Lucia MS, Thompson IM, Ford LG, et al. Effect of selenium and vitamin $\mathrm{E}$ on risk of prostate cancer and other cancers: the Selenium and Vitamin E Cancer Prevention Trial (SELECT). JAMA 2009; 301:39-51.

10. Secretaria Nacional de Assistência à Saúde, Ministério da Saúde/Instituto Nacional de Câncer. Câncer de próstata: consenso. Rio de Janeiro: Instituto Nacional de Câncer; 2002.

11. Nagler HM, Gerber EW, Homel P, Wagner JR, Norton J, Lebovitch S, et al. Digital rectal examination is barrier to population-based prostate cancer screening. Urology 2005; 65:1137-40. 
12. Thompson IM, Ankerst D. Prostate - specific antigen in the early detection of prostate cancer. CMAJ 2007; 176:1853-8.

13. Wolf AM, Wender RC, Etzioni RB, Thompson IM, D'Amico AV, Volk RJ, et al. American Cancer Society guideline for the early detection of prostate cancer: update 2010. CA Cancer J Clin 2010; 60:70-98.

14. Schröder FH, Hugosson J, Roobol MJ, Tammela TL, Ciatto S, Nelen V, et al. Screening and prostatecancer mortality in a randomized European study. N Engl J Med 2009; 360:1320-8.

15. Andriole GL, Crawford ED, Grubb 3rd RL, Byus SS, Chia D, Church TR, et al. Mortality results from a randomized prostate-cancer screening trial. $\mathrm{N}$ Engl J Med 2009; 360:1310-9.

16. Djulbegovic M, Beyth RJ, Neuberger MM, Stoffs TL, Vieweg J, Djulbegovic B, et al. Screening for prostate cancer: systematic review and metaanalysis of randomised controlled trials. BMJ 2010; 341:c4543.

17. Preventive Services Task Force. Screening for prostate cancer. http://www.uspreventiveservicestask force.org/uspstf/uspsprca.htm (acessado em 29/ Set/2010).

18. American Cancer Society. American Cancer Society recommendations for prostate cancer early detection. http://www.cancer.org/Cancer/Pros tateCancer/MoreInformation/ProstateCancerEar lyDetection/prostate-cancer-early-detection-a-cs-recommendations (acessado em 29/Set/2010).

19. Lin K, Lipsitz R, Miller T, Janakiraman S. Benefits and harms of prostate-specific antigen screening for prostate cancer: an evidence update for the U.S. Preventive Services Task Force. Ann Intern Med 2008; 149:192-9.

20. Instituto Nacional de Câncer. INCA esclarece população sobre rastreamento do câncer de próstata. http://www.inca.gov.br/releases/press_release view.asp?ID=1967 (acessado em 19/Nov/2008).

21. Lima-Costa MFF. A saúde dos adultos na região metropolitana de Belo Horizonte: um estudo epidemiológico de base populacional. Belo Horizonte: Núcleo de Estudos em Saúde Pública e Envelhecimento, Fundação Oswaldo Cruz/Universidade Federal de Minas Gerais; 2004.

22. Rutten LJF, Meissner HI, Breen N, Vernon SW, Rimer BK. Factors associated with men's use of prostate-specific antigen screening: evidence from Health Information National Trends Survey. Prev Med 2005; 40:461-8.

23. Spencer BA, Babey SH, Etzioni DA, Ponce NA, Brown ER, Yu H, et al. A population-based survey of prostate - specific antigen testing among California men at higher risk for prostate carcinoma. Cancer 2006; 106:765-74.

24. Ross LE, Coates RJ, Breen N, Uhler RJ, Potosky AL, Blackman D. Prostate-specific antigen test use reported in the 2000 National Health Interview Survey. Prev Med 2004; 38:732-44

25. Ross LE, Berkowitz Z, Ekwueme DU. Use of the prostate-specific antigen test among U.S. men: findings from the 2005 National Health Interview Survey. Cancer Epidemiol Biomarkers Prev 2008; 17:636-44.
26. Fowker JH, Schlundt D, Signorello LB, Ukoli FAM, Blot WJ. Prostate cancer screening between lowincome African-American and Caucasian men. Urol Oncol 2005; 23:333-40.

27. César LGC, Carandina L, Alves MCGP, Barros MBA Goldbaum M. Saúde e condição de vida em São Paulo. Inquérito Multicêntrico de Saúde no Estado de São Paulo - ISA-SP. São Paulo: Faculdade de Saúde Pública, Universidade de São Paulo; 2005.

28. Mayfield D, McLeod G, Hall P. The CAGE questionnaire: validation of new alcoholism screening instrument. Am J Psychiatry 1974; 131:1121-3.

29. Mari JJ, Williams P. A validity study of a psychiatric screening questionnaire (SRQ 20) in primary care in the city of São Paulo. Br J Psychiatry 1986; 148:23-6.

30. Grönberg H. Prostate cancer epidemiology. Lancet 2003; 361:859-64.

31. Antonopoulos IM, Pompeo ACL, El Hayek OR, Sarkis AS, Alfer Junior W, Arap S. Results of prostate cancer screening in non-symptomatic men. Braz J Urol 2001; 27:227-34.

32. Berger AP, Deilb M, Halpern EJ, Lechleitner M, Bektic J, Horninger W, et al. Vascular damage induced by type 2 diabetes mellitus as a risk factor for benign prostatic hyperplasia. Diabetologia 2005; 48:784-9.

33. Bonovas S, Filioussi K, Tsantes A. Diabetes mellitus and risk of prostate cancer: a meta-analysis. Diabetologia 2004; 47:1071-8.

34. Kasper JS, Giovannucci E. A meta-analysis of diabetes mellitus and the risk of prostate cancer. Cancer Epidemiol Biomarkers Prev 2006; 15:2056-62.

35. Ramirez A, Farmer GC, Grant D, Papachristou T. Disability and preventive cancer screening: results from the 2001 California Health Interview Survey. Am J Public Health 2005; 95:2057-64.

36. Norcross W, Ramirez C, Palinkas L. The influence of women on the health care-seeking of men. J Fam Pract 1996; 43:475-80.

37. Volk RJ, Cass AR. The accuracy of primary care patients self-reports of prostate-specific antigen testing. Am J Prev Med 2002; 22:56-9.

38. Hall HI, Eeden SKVD, Tolsma DD, Rardin K, Thompson T, Sinclair AH, et al. Testing for prostate and colorectal cancer: comparison of self report and medical record audit. Prev Med 2004; 39:27-35.

39. Rauscher GH, Johnson TP, Cho YI, Walk JA. Accuracy of self-reported cancer-screening histories: a meta-analysis. Cancer Epidemiol Biomarkers Prev 2008; 17:748-75.

40. Departamento de Ações Programáticas e Estratégicas, Secretaria de Atenção à Saúde, Ministério da Saúde. Política Nacional de Atenção Integral à Saúde do Homem. Princípios e diretrizes. Brasília: Ministério da Saúde; 2008.

Recebido em 06/Out/2009

Versão final reapresentada em 20/Out/2010

Aprovado em 23/Dez/2010 